\title{
Computational biomechanics of the cardiovascular system
}

\author{
T. Yamaguchi \\ Department of Bioengineering and Robotics, School of Engineering, \\ Tohoku University, Sendai, Japan
}

\begin{abstract}
Due to rapid advances in invasive and non-invasive imaging technology in clinical medicine, it is now possible to obtain images of target organs for use in computational analysis modelling. Our ultimate goal is to build a system that can assist clinicians in diagnosis, treatment planning, and follow-up of patients with cardiovascular diseases, and as patients differ in terms of anatomical configuration and disease condition, a wide variety of patient data must be accumulated, not only for statistical analysis but also to improve the processing system. Therefore, a comprehensive database that includes patient data, medical images, computational models, and computed results must be constructed that is within the scope of our current projects. This is part of a long-term research program in the field of computational medicine, which will inevitably become part of mainstream medical development in the near future. The introduction of computational mechanical assistance to medical diagnosis and treatment will enhance the objectiveness of medical decisions.
\end{abstract}

Keywords: computational study, cardiovascular system, clinical application, medical image, blood flow-vascular wall interactions, atherosclerosis.

\section{Introduction}

In the past decades, a great advance was achieved in the computer technology and it enabled us to analyze various phenomena that were not possible to study by numerical means before. Complex biological subjects are nothing exceptional. One of the most successful fields among those conducted by computational methods was the study of the blood flow. Many investigators including us started from fairly simple objects to quite complicated system and 
widened the horizon of the computational research not only for fundamental understandings but also for direct or indirect clinical applications.

Cardiovascular diseases are the most important causes of deaths in Western as well as Eastern industrialized countries with an increase of elderly population and the change of dietary habit of candidate peoples belong to younger generations.

The most significant background condition of the cardiovascular diseases is the atherosclerosis. It causes the myocardial infarction when it occurs in the coronary artery of the heart and the various types of cerebral strokes when it affects the arteries in the brain. The atherosclerosis is not a simple disease as generally presumed. An accumulation of lipid in the arterial wall occurs where some precursory or early changes of the arterial wall cellular structure are formed under an influence of the mechanical circumstances of the arterial wall including the wall shear stress.

To determine the mechanical environments in the blood vessels, we encounter various difficulties. They are, firstly, the complex geometry of the vascular system. It is no longer a simple tubular system with a circular cross section, but it is with curves, torsions, branches, tapers, etc with individually different topological (i.e. connections of branches) structure. The vascular system differs from one person to other just like as their faces. Secondly, we always have to analyze it under unsteady conditions due to pulsating motion of the heart, the only energy source in the cardiovascular system. Thirdly, the wall of the artery is not rigid, and this imposes serious difficulty when it is combined with the unsteady flows. Fourthly, the blood, the working fluid of the cardiovascular system, is never a Newtonian fluid, since it consists of substantial volume fraction of cellular components such as the red blood cells.

All these problems are nonlinear and difficult to deal with by experimental means in the real blood flow. This is particularly true when they are to be examined in a human body. Restriction of the measurements in the real human body is very strong and the computational fluid dynamics (CFD) method is, in a sense, the only solution to analyze the blood flow phenomena.

\section{Aim of the development}

It is therefore necessary to establish the methods to capture and analyze the complex nature of the interactions among the blood constituents, the blood flow and the vessel wall. We have been developing an integrated system for the blood flow analysis using computational fluid mechanics based on various imaging technologies that are widely used in the clinical practice of medicine (Mori et al [1,2], Nakamura et al [3], Feng et al [4], Rathish Kumar et al [5]). We are developing this system for its possible use in an emergency medical diagnosis and treatments by supporting the clinical decision made by medical practitioners, providing more quantitative and predictive information.

In the course of the development, we could complete at least the first comprehensive software system that can be implemented in the real medical practice. It is expected to be the first usable system as such in the field of CFD 
applied to the clinical medicine. By using the currently proposed methods, we would like to show the potential of the developed system.

\section{Developed system}

\subsection{Use case and general user interface}

As a primary use case, we postulated a scenario of a semi-emergency case of cardiovascular events such as suspected rupture of the aortic aneurysm and an acute coronary syndrome. The scenario starts with an admittance of a patient whose history is not well known but the clinical condition strongly suggest acute and urgent cardiovascular events. In such a case, some urgent examinations, including CT or MRI, are usually conducted. We assume that there is no sufficient time for computations before the clinical decision is made.

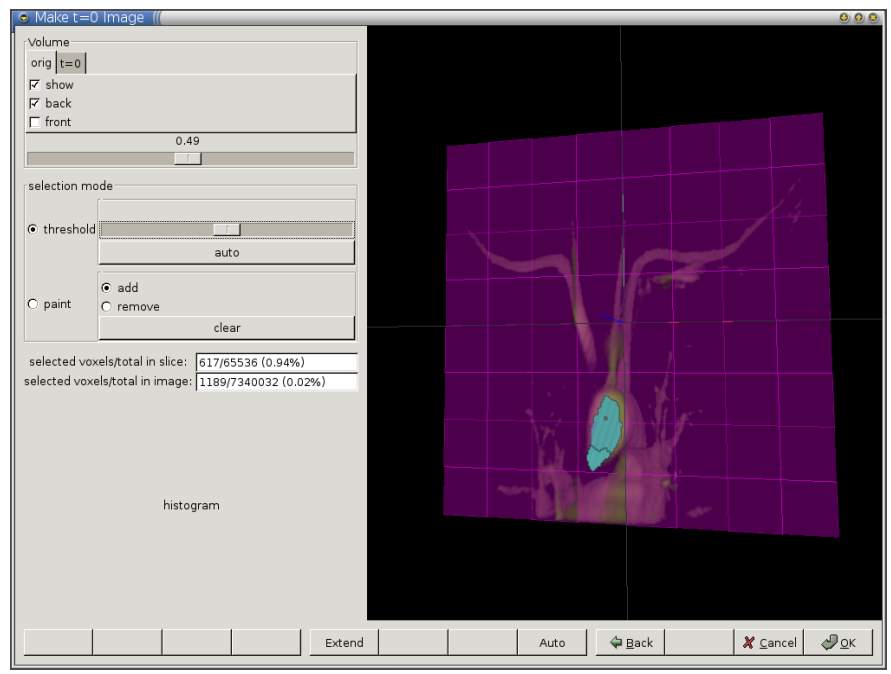

Figure 1: An interface screen for medical volume image handling to check the threshold of image registration.

\subsection{Handling of medical images}

Volumetric representations of the clinical images should be constructed on line with imaging instruments through network connections such as DICOM formatted communications. It is necessary to handle the volumetric data reconstructed from slices of tomographic images as fast as possible to enable a user to comprehend the problem. Our system can handle those data by using a very fast volume rendering method utilizing recently available graphics facility implemented in PC class computers (Fig. 1). 


\subsection{Characterization of the structure}

Our fundamental idea of designing whole system of the CFD tools is that we rely on human ability of pattern recognition in searching and characterizing structures in complex medical images. We, therefore, designed everything, including registration, segmentation, and model building to be performed in a semiautomatic manner, in which the human decision is always of a paramount value. Operators, through sophisticated interfaces, can direct how to, where to, and what to be done for necessary procedures. For example when a structure such as an aorta is segmented from the raw image, a user can direct where it starts, ends, and passes, manually so that no erroneous automatic differentiation is necessary (Fig.2).

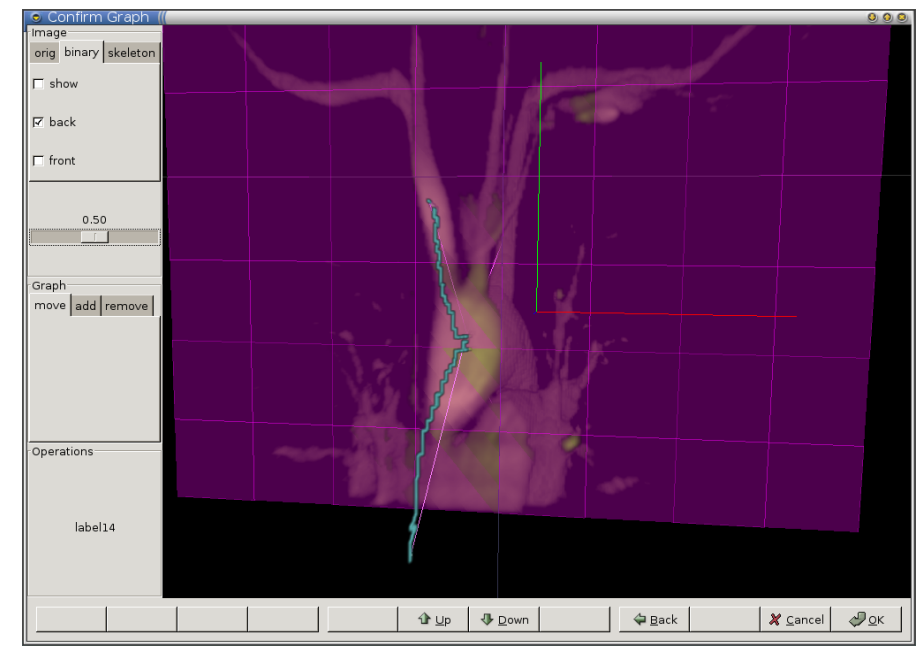

Figure 2: An interface for extracting graph structure of the branching arteries.

\subsection{CFD model building}

The arterial systems were, as the first order approximation, represented a combined pipes separately modeled. The component pipes were connected each other by using an overset grid method in which every component shares some portion of itself with other components. Physical properties such as the velocity and pressure are exchanged between those overlapped portions of the components when the computational step proceeds to the next iterative step. We also developed an interface system to modify fine structures and surfaces of the components so that the shape of the model can be fitted onto the measured real surface of corresponding medical images. 


\subsection{Database of combined images, models and computed results indexed by the structure}

A novel indexing system was devised so that the connectivity or the skeleton representation of complex geometry of the arterial system can be semiautomatically extracted from 3D volume rendered images of cardiovascular system. Varieties of graphical representations are used as index keys of related real images, computational models, and pre-computed results using the model. Once a new urgent patient comes to the clinic, a set of MRI or CT are taken and the extracted features will be used to retrieve the set of images, models, and precomputed results by using its skeleton as a key of database. If the patient is in urgent condition, the pre-computed results modified according to the difference of the model and the real patient can be used as the first order approximation of the blood flow estimation. Though we are fully aware of non-linearity of the flow phenomenon, it would be much better to have some means to imagine what is happening in the patient using pre-computed results rather than having nothing but just an empirical sometimes illogical imagination. By establishing this system, we will easily built a large-scale database of normal and diseased human cardiovascular system (Fig.3).

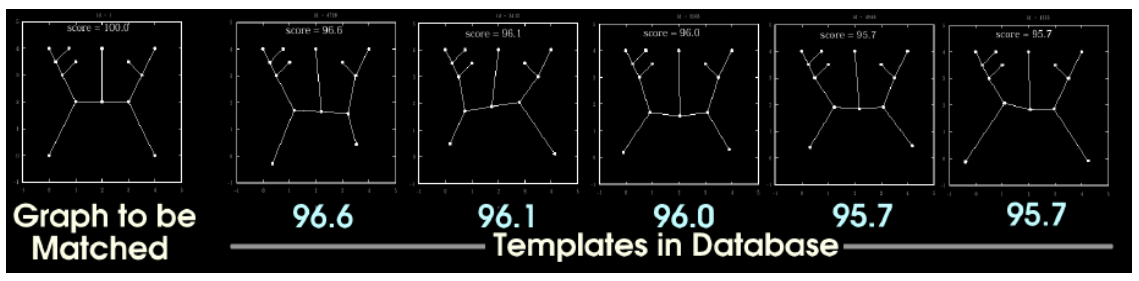

Figure 3: Matching of patterns of branching of aorta and its large branches. Scores were automatically computed using the graph theory.

\subsection{Visualization and feed back to the clinical medicine}

Visualization program with a facility of 4D virtual reality interface is equipped in the present system. Various types of visualization are implemented so that distribution patterns of necessary physical parameters over the cardiovascular system is examined with respect to the configuration, physiological conditions and pathological states of the system to predict the outcome of the present diseases and to plan the most appropriate therapeutic measures based on the computed results.

\section{Results and discussion}

We could establish a new integrated software system for the application of the computational mechanics to the cardiovascular clinical medicine. The developed system is with facilities of handling medical images, extract of basic structures, building of computational models, and comprehensive database indexed by the 
structure of the cardiovascular system expressed in terms of the graphical representation. The developed system is now going to be applied to the real clinical practice to accumulate the real data of patients.

We distinguish four major problems with respect to computational methodology, and recognize three biological necessities in advancing computational biomechanical analysis of the cardiovascular system. Computational difficulties are; firstly, complex and individually different geometry of the cardiovascular system, particularly the vasculature. Secondly, flexible wall of very complex nonlinear material properties of the whole cardiovascular conduit should be pointed out. Thirdly, the pulsatile nature of the blood flow imposes very complex interactions with the former two characteristics, and fourthly, the non-Newtonian viscous nature of the blood is not a simple matter of material properties. They are all individually hard targets in terms of computational mechanics, and even more difficult when they interacts together to form the natural blood flow in the real cardiovascular system. Since we started to apply, firstly, the computational fluid dynamics (CFD), and later the computational mechanics (fluid and solid interactions) to the cardiovascular system, they continue to be the major problems in the computational method.

In addition to the technological tasks above, it is necessary to pay full attention to biological complexities in the analysis of the blood flow, especially with respect to the disease process. Most of modern analysis of the cardiovascular system is strongly motivated by some pathological processes. Among those, the atherosclerosis has been and still is the most important disease in terms of mechanical analysis of the blood flow. Together with other vascular disorders, including the cerebral and aortic aneurysm, and thrombotic diseases, atherosclerosis is a typically chronic disease, which takes decades of life to initiate, to develop, and to become lethal in the latter half of the life. It means that the whole process of the disease, from the initiation to the last fatal stage is under the influence of biological responses and adaptations of the living system. The target of our study is not merely an artifact, which is manufactured by mankind and is not an actively responding system once they are installed and just to be degraded. Most of so-called CFD studies, unfortunately, including our past studies, did not keep this point in mind. While the living system, either as a whole, or in their subsystems, such as the cardiovascular system, is always under integrated control of the nervous and humoral control of the whole body, in other words, in the homeostasis. The control of the whole body and organs is in a truly integrated manner in which multiple feedbacks of mutual interactions are implemented. These control mechanisms are with different coverage and time constants, from micro-scale to macro-scale, and from nanosecond to decades. Moreover, it is gradually understood that the living system per se can only be understood in the light of its historical, in other words, evolutionary process. This point may not be distinctive when we analyze idealized vascular system, but will be crucial when we are to understand and to locate total physiological and pathological processes in the whole living system. 


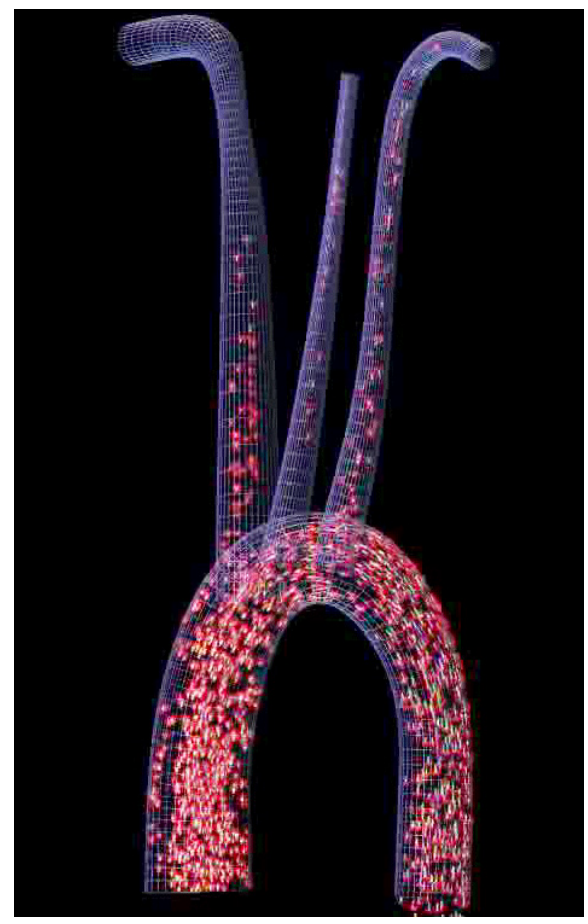

Figure 4: Visualized blood flow in a model constructed by using the current overset mesh method.

\section{Acknowledgements}

Support of Tohoku University 21 COE Program "Future Medical Engineering based on Bio-nanotechnology" is gratefully acknowledged. Part of these studies was supported by Grant-in-Aid's for Scientific Research from the Ministry of Education, Culture, Sports, Science and Technology of Japan \#16200031, \#15086204, and \#17636005.

\section{References}

[1] Mori D. \& Yamaguchi T., Computational Fluid Dynamics Modeling and Analysis of the Effect of 3-D Distortion of the Human Aortic Arch Computer Methods in BIomechanics and Biomedical Engineering 5, pp. 249-260, 2002.

[2] Mori D., Hayasaka T. \& Yamaguchi T., Modeling of the Human Aortic Arch with Its Major Branches for Computational Fluid Dynamics Simulation of the Blood Flow. JSME International Journal Series C 45 pp. 997-1002, 2002. 
[3] Nakamura M., Wada S., Mikami T., Kitabatake A., Karino T. \& Yamaguchi T., Effect of flow disturbances remaining at the beginning of diastole on intraventricular diastolic flow and colour M-mode Doppler echocardiograms. Medical \& Biological Engineering \& Computing, 42 pp.509-515, 2004.

[4] Feng Y., Wada S., Tsubota K. \& Yamaguchi T., Growth of Intracranial Aneurysms Arised from Curved Vessels under the Influence of Elevated Wall Shear Stress - A Computer Simulation Study, JSME International Journal, Series C, 47 pp. 1035-1042, 2004.

[5] Rathish KUMAR B. V., Yamaguchi T., Liu H. \& Himeno R, Blood Flow in a Vessel with Asymmetric Aneurysm, International Journal of Applied Mechanics and Engineering, 9 pp. 505-521,2004. 